\title{
Une nouvelle découverte d'art pariétal aurignacien in situ à l'abri Castanet (Dordogne, France) : contexte et datation
}

Romain Mensan, Raphaëlle Bourrillon, Catherine Cretin, Randall White, Philippe Gardère, Laurent Chiotti, Matthew Sisk, Amy Clark, Thomas Higham et Élise Tartar

\section{(2) OpenEdition}

\section{Journals}

Édition électronique

URL : http://journals.openedition.org/paleo/2356

DOI : $10.4000 /$ paleo.2356

ISSN : $2101-0420$

Éditeur

SAMRA

Édition imprimée

Date de publication : 15 décembre 2012

Pagination : 171-188

ISSN : $1145-3370$

\section{Référence électronique}

Romain Mensan, Raphaëlle Bourrillon, Catherine Cretin, Randall White, Philippe Gardère, Laurent Chiotti, Matthew Sisk, Amy Clark, Thomas Higham et Élise Tartar, « Une nouvelle découverte d'art pariétal aurignacien in situ à l'abri Castanet (Dordogne, France) : contexte et datation », PALEO [En ligne], 23 | 2012, mis en ligne le 19 avril 2013, consulté le 25 juillet 2020. URL : http:// journals.openedition.org/paleo/2356; DOI : https://doi.org/10.4000/paleo.2356

Ce document a été généré automatiquement le 25 juillet 2020.

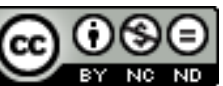

PALEO est mis à disposition selon les termes de la licence Creative Commons Attribution - Pas d'Utilisation Commerciale - Pas de Modification 4.0 International. 


\title{
Une nouvelle découverte d'art pariétal aurignacien in situ à l'abri Castanet (Dordogne, France) : contexte et datation
}

\author{
Romain Mensan, Raphaëlle Bourrillon, Catherine Cretin, Randall White, \\ Philippe Gardère, Laurent Chiotti, Matthew Sisk, Amy Clark, Thomas \\ Higham et Élise Tartar
}

\section{1 - Introduction}

1 Cet article présente l'exceptionnelle découverte d'un fragment de voûte ornée, en juillet 2007 sur le site aurignacien ancien de l'abri Castanet (commune de Sergeac, Dordogne). Depuis la découverte, l'équipe a entrepris une vaste réflexion pluridisciplinaire destinée à l'étude et à la contextualisation du bloc au sein des remplissages de l'abri Castanet, mais aussi à une échelle beaucoup plus large, celle du vallon de Castel-Merle. Il s'agit de replacer l'expression graphique des premiers Hommes modernes d'Europe occidentale avec l'ensemble des activités humaines, quotidiennes ou non, exercées sur le site.

2 Après une présentation générale des problématiques archéologiques inhérentes à la période et au site étudié, les objectifs initiaux ayant présidé à une relecture de la coupe laissée par D. Peyrony en 1925 seront précisés, ainsi que les circonstances exactes de la découverte du bloc et le choix de la méthode utilisée pour son extraction.

3 Une description du bloc lui-même, des techniques utilisées pour son étude des entités graphiques qu'il possède sera ensuite proposée.

4 Enfin, une réflexion pluridisciplinaire relative à la mise en relation de cette découverte avec le reste des données (archéologiques et environnementales) sera détaillée. 


\section{2 - Contexte historique et de découverte}

5 L'abri Castanet est l'un des nombreux gisements sous abri du vallon de Castel-Merle, sur la commune de Sergeac, débouchant sur la rive gauche de la Vézère, entre Les Eyzies et Montignac (fig. 1). Le site fut découvert en 1911 par M. Castanet sur le versant est du vallon, alors qu'il travaillait pour L. Didon comme ouvrier de fouille à l'abri Blanchard, situé plus au nord (White et al. 2012). Les fouilles ont été entreprises alors par M. Castanet sous la supervision de D. Peyrony de 1911 à 1913, puis en 1924-1925.

\section{Les fouilles anciennes}

6 Le site est un abri sous-roche effondré orienté vers l'ouest, qui a livré un riche assemblage réparti en deux niveaux archéologiques, l'un et l'autre attribuables à l'Aurignacien (fig. 2). D. Peyrony ne publia ses résultats sur l'abri Castanet qu'en 1935 (Peyrony 1935). Il distingua un niveau inférieur reposant directement sur le socle calcaire et un niveau supérieur très pauvre, séparé du premier par une couche stérile, le tout étant recouvert par l'effondrement de l'abri. Le niveau inférieur est attribué à l'Aurignacien ancien (Aurignacien I) en raison de ses nombreuses pointes en bois de cervidé à base fendue, et le niveau supérieur à l'Aurignacien récent (Aurignacien II) sur la base de la présence de deux pointes en os à base massive. Cependant, l'industrie lithique des deux niveaux apparaît identique, comme l'a démontré D. de SonnevilleBordes en 1960 lors de l'étude de la série Peyrony (Sonneville-Bordes 1960).

7 De même que l'abri Blanchard, l'abri Castanet se révéla très riche en éléments de parure (White 2008b), en industrie osseuse (Tartar 2007, 2009), mais également en manifestations anthropiques sur support rocheux (art pariétal, art sur bloc, anneaux façonnés...). Cependant, même si M. Castanet attribue des supports gravés ou peints à certaines couches, constatant d'ailleurs régulièrement que leur surface décorée était orientée vers le sol, aucun ne bénéficie d'un véritable contexte archéologique. La plupart d'entre eux ont été retrouvés après la fouille, parmi les blocs mis de côté pour inspection et lavage.

Bien que de nombreux sites aurignaciens aient été fouillés depuis une quarantaine d'années dans le sud-ouest de la France (La Ferrassie, Le Flageolet, Les Rois, Brassempouy, l'abri Pataud, Le Piage, etc. $)^{1}$, aucun n'apparait comparable à l'ensemble Castanet-Blanchard, notamment en ce qui concerne de tels témoignages rupestres. L'évolution des questions théoriques et des méthodes archéologiques rendait donc intéressante la reprise d'un projet de fouille à l'abri Castanet. 
Figure 1 - Situation géographique de l'abri-sous-roche de Castanet dans le Vallon des Roches à Sergeac (Dordogne, France), points rouges : principaux sites Aurignaciens des vallées Vézère et Dordogne.

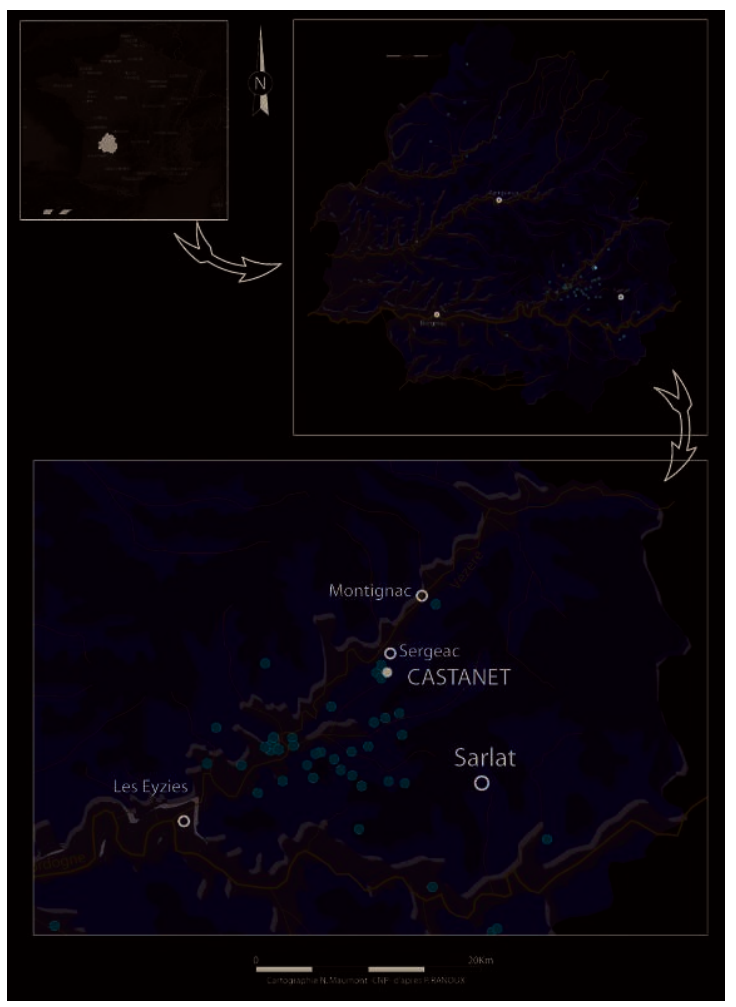

Figure 2 - Abris Castanet et Blanchard (Sergeac, Dordogne), vestiges archéologiques représentatifs de la culture aurignacienne. $1:$ Industrie osseuse ; 2 : Industrie lithique ; $3:$ Parure ; $4:$ Art pariétal.

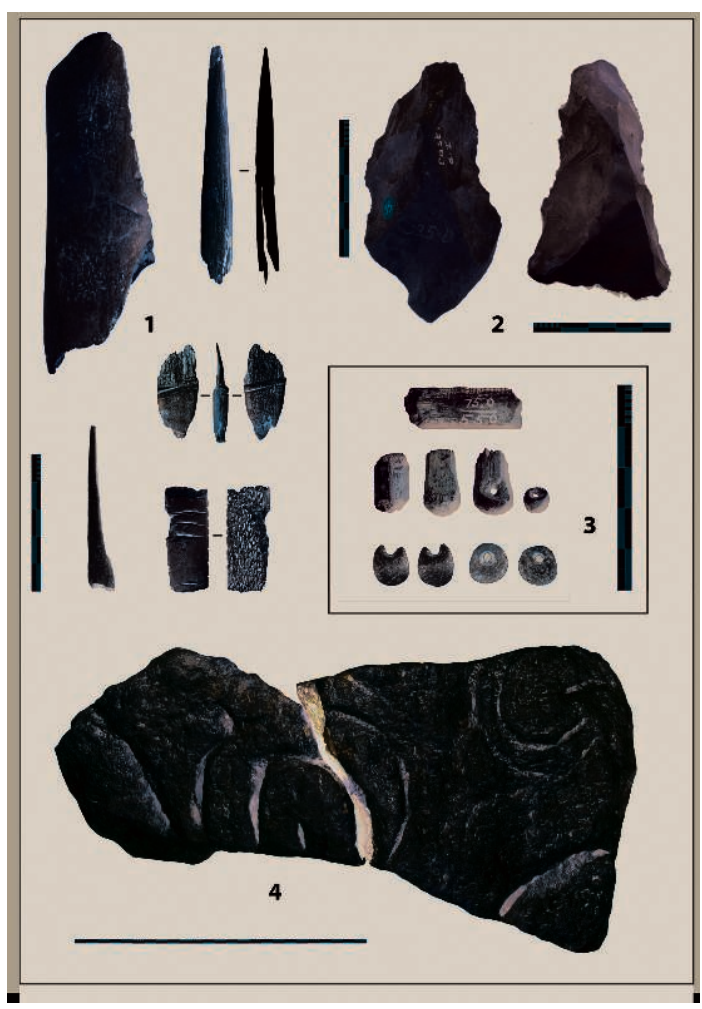




\section{Les fouilles récentes}

9 Une opération de diagnostic fut donc lancée en 1994 par J. Pelegrin et R. White (Pelegrin et White 1998). Les principaux objectifs étaient de reconnaître l'emplacement de la fouille de D. Peyrony et M. Castanet afin d'y chercher d'éventuels dépôts en place sous les déblais, de mettre au propre une coupe sagittale perceptible dans le talus et, enfin, d'évaluer le potentiel archéologique restant plus au sud.

10 Les dépôts rencontrés dans le secteur de l'ancienne fouille Peyrony constituent sans ambiguïté les déblais des fouilles anciennes et n'ont livré aucun niveau en place. Seul le nettoyage de la coupe sagittale a permis de retrouver le niveau aurignacien au contact du socle calcaire. Plusieurs sondages effectués dans le talus au sud du secteur Peyrony ont en revanche permis de confirmer la présence de ce même niveau à près de dix mètres au sud de la coupe laissée par D. Peyrony. Une opération de fouille pluriannuelle a donc été entreprise de 1995 à 1998, dans le secteur maintenant dénommé « secteur sud». Ces premières fouilles modernes dans le vallon des Roches ont révélé une stratigraphie relativement simple :

- un niveau archéologique de base localisé directement sur le socle ;

- effondrement de l'auvent ;

- un colmatage par apports sédimentaires depuis le plateau.

11 Les Hommes préhistoriques s'installèrent donc directement sur le plancher calcaire de l'abri. Protégés sous un porche, ils occupèrent une surface large de quelques mètres avant que l'effondrement de l'auvent scelle les dépôts anthropiques. Sur cet éboulis, s'est stabilisée ensuite une épaisse couche de sédiments, formant ainsi un large cône appuyé contre la paroi rocheuse. Les opérations de 1994 à 1998 ont contribué à une bonne compréhension du remplissage de l'abri et ont mis en évidence des phénomènes pré- et post-dépositionnels ayant pu perturber le remplissage.

Précisons que le niveau supérieur attribué à l'Aurignacien récent (Aurignacien II) par D. Peyrony a été mis en doute lors de ces premiers diagnostics, aucune couche postérieure à celle située au contact du bed-rock n'ayant été retrouvée.

\section{Contexte de la découverte}

13 Les investigations dans le secteur sud de l'abri ont repris depuis 2005 (White 2005, 2006, 2007a, 2008a, 2009a ; White et al. 2010). Les objectifs étaient de documenter sur une plus grande surface le niveau Aurignacien, mais également le replacer dans son contexte géomorphologique (Mensan 2007; Mensan et Sisk 2008). Pour ce faire, une étude pluridisciplinaire a été engagée dans tout le vallon afin d'intégrer les données historiographiques, géologiques, géomorphologiques, topographiques et archéologiques dans une réflexion globale sur les occupations du versant oriental du vallon des Roches.

Cette nouvelle approche a permis de poursuivre la fouille du secteur sud de l'abri et d'intégrer dans notre projet le secteur nord, dit "secteur Peyrony ", afin d'observer le niveau archéologique identifié dans la coupe sagittale et de le comparer à celui du secteur sud. Le relevé stratigraphique, réalisé en 2006, montre que ce niveau se situe directement au contact du socle, position identique à celle observée au sud. Déjà identifié en 1994, ce niveau avait été dénommé " purple layer ", il est caractérisé par une très grande richesse en mobilier et par sa coloration pourpre. Dans le cadre de la 
caractérisation des variations latérales de faciès d'un niveau archéologique, la coupe sagittale laissée par Peyrony constitue une fenêtre appropriée à l'observation à longue distance de ces phénomènes.

15 Le secteur nord présente une stratigraphie globalement identique à celle observée dans le secteur sud. Cependant, à l'inverse du secteur sud où seule la partie arrière du remplissage de l'abri est conservée, le secteur Peyrony représente la zone avant de celui-ci. Cette même zone avant est actuellement absente au sud : elle fut détruite lors de l'aménagement de la route dans les années soixante.

Sur la petite fenêtre d'observation étudiée, le niveau archéologique était notamment scellé par un bloc qui semblait provenir de l'effondrement du porche de l'abri, luimême surmonté par les niveaux supérieurs de comblement. Il était donc nécessaire de l'enlever. Ce bloc rocheux se trouvait enchâssé dans la coupe abandonnée par Peyrony en 1925. Il dépassait du témoin stratigraphique, ses dimensions ayant dû empêcher son évacuation. Une partie a d'ailleurs été coupée anciennement, sans doute à l'époque des fouilles de 1924-1925.

17 Cette coupe a été relevée en 1995 (Texier 2009 - p. 105) et reprise en 2006 (White 2006) (fig. 3) mais il ne s'agit vraisemblablement pas de la coupe relevée par Peyrony qui serait située plusieurs mètres au nord de celle-ci. Ce bloc, que nous avons dénommé «bloc $\mathrm{K}$ » lors du relevé effectué en 2006, était déjà bien visible lors de l'opération de 1994 mais son volume exact était alors inconnu, puisqu'il pénétrait à l'intérieur du remplissage. A posteriori, il s'avère que celui-ci fait environ $0,650 \mathrm{~m} 3$ et pèse plus d'une $1,5 \mathrm{t}$.

Figure 3 - À gauche, relevé stratigraphique de D. Peyrony ; à droite, stratigraphie effectuée en 2007.

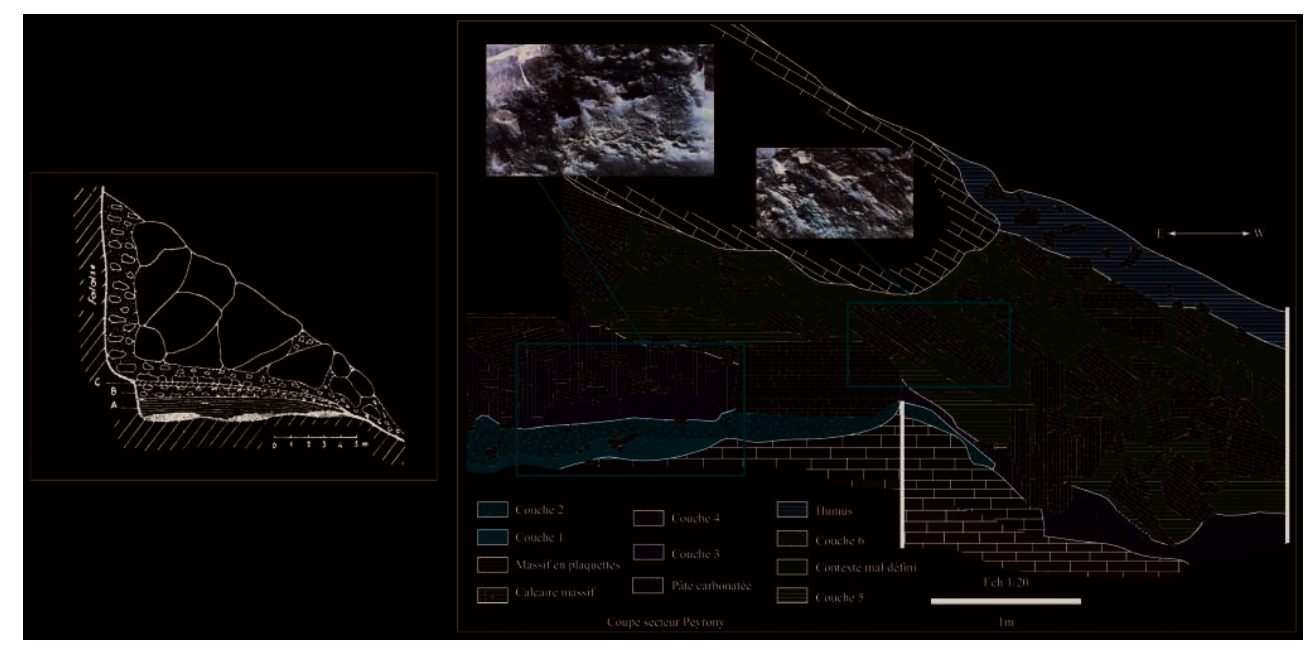


Figure 4 - Haut, coupe Peyrony avec le bloc K en place ; bas, coupe Peyrony une fois le bloc K extrait.

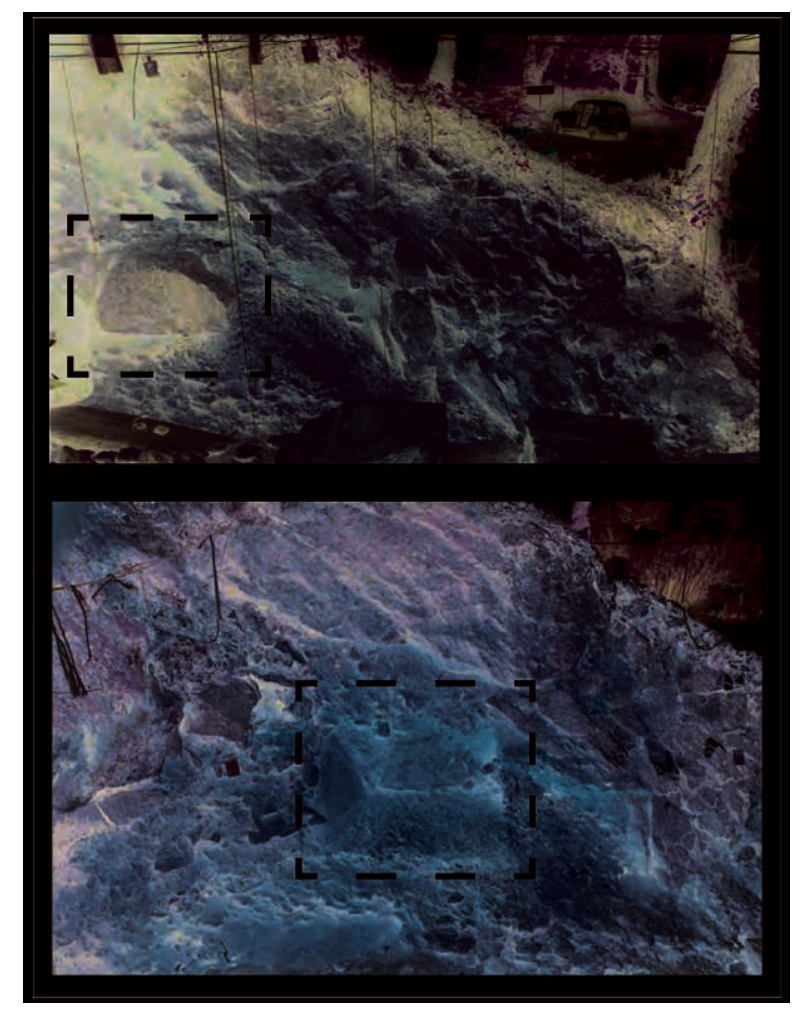

\section{Du prélèvement du bloc $\mathrm{K}$ à la découverte des éléments graphiques : contexte, méthode, restitution}

\section{Méthode d'extraction}

L'objectif était d'extraire un bloc de dimension alors inconnue sans perturber ni le niveau archéologique sous-jacent ni la coupe, laquelle était coiffée d'un énorme pan rocheux (White et al. 2007) (fig. 4). La volonté de préserver la couche archéologique sous jacente résulte de la réflexion engagée dès la reprise du secteur sud en 2005, pour caractériser des variations latérales de faciès sur un niveau en même position stratigraphique dans le vallon. La position du témoin stratigraphique, au fond d'une tranchée étroite, en haut d'un talus de plus $1,5 \mathrm{~m}$ de haut, ne permettait pas l'utilisation de moyens de levage mécanique qui, de toute façon, auraient nécessité l'enlèvement de la masse rocheuse sus-jacente, entraîné l'arrachement brutal d'une grande quantité de sédiments et empêché l'étude fine des relations entre le bloc, la couche archéologique et les remplissages sus-jacents (archéologiques ou non).

L'option retenue a été de le subdiviser en éléments maniables à l'aide d'un perforateur et de coins éclateurs de carriers. L'éventualité de la présence d'un décor sur le bloc a bien sûr été envisagée dès le début de l'opération et la manipulation du bloc fragmenté paraissait préférable à la traction mécanique du fait de l'impossibilité de faire la moindre vérification au cours de l'enlèvement si tel avait été l'option retenue. Ce procédé a permis de maîtriser parfaitement le découpage du bloc qui au final a pu être restitué dans toute son intégrité. De plus, la prise systématique de photographie à chaque étape $\mathrm{du}$ prélèvement s'est avérée extrêmement précieuse pour la 
contextualisation du bloc orné. Après aménagement du secteur par une plateforme audessus de la tranchée, il a été convenu de soulever les fragments du bloc progressivement, de vérifier que la couche sous-jacente n'adhérait pas aux fragments, puis de les faire doucement basculer sur un côté en les calant systématiquement sur des sacs de sable afin d'assurer leur stabilité : les surfaces en contact avec la couche archéologique n'ont donc jamais été touchées, ni lavées.

Entre le 22 juin et le 09 juillet 2007, ce sont au total douze fragments qui ont été détachés à partir du bloc $\mathrm{K}$. Sept d'entre eux, (K1 et 7), ont emporté une partie de la face ornée, en contact avec la couche archéologique. Dans le prolongement de ce bloc $\mathrm{K}$ et dans l'épaisseur de la coupe, vers le sud, une quinzaine d'autres blocs appartenant manifestement à la même masse rocheuse originelle, cassés en place, soit au moment de leur chute, soit par compression, ont été prélevés les 12 et 13 juillet 2007.

\section{Restitution du bloc après subdivision}

21 Il est important de garder en mémoire que la numérotation a été réalisée a posteriori à partir de l'observation de la surface ornée, en l'occurence la face inférieure du bloc lorsque celui-ci se trouvait en place. Il existe donc une discontinuité entre la chronologie des opérations de terrain et cette numérotation ${ }^{2}$ (fig. 5).

\section{3 - Les éléments graphiques : premières observations}

\section{Techniques d'étude du bloc}

Malgré toutes les précautions prises afin de conserver l'intégrité de la couche pourpre sous-jacente, la face inférieure du bloc était tout de même recouverte d'une fine pellicule de sédiments (jusqu'à $1 \mathrm{~cm}$ ) ne permettant pas de lire avec précision l'état de surface, les traces techniques et les vestiges graphiques. Dans un souci de préservation et de conservation du bloc, aucun nettoyage n'a été effectué après son extraction (Bourrillon 2007; White et Bourrillon 2008). Dans ce sédiment, des fragments d'os et des éclats de silex de petite dimension sont encore en contact avec la face ornée du bloc. Dans l'attente du nettoyage de la surface, il s'agit donc ici d'un déchiffrage superficiel dont les propositions interprétatives - technologiques et iconographiques seront donc à confirmer ou à infirmer.

Afin de déterminer la présence éventuelle de pigments anthropiques et de mesurer l'impact d'un nettoyage superficiel de la surface, il a été fait appel à des analyses élémentaires : des mesures de fluorescence $\mathrm{X}$ ont été réalisées au cours de la dernière campagne, en 2010, par J. de Sanoit et D. Chambellan du CEA (Sanoit (de) et al. 2005). Les premiers résultats des analyses confirment la stabilité de la coloration, ce qui permet d'envisager à terme un nettoyage superficiel au pinceau sec de la surface ornée. Précisons que la composition de la coloration rouge présente sur le bloc, n'est pas distinguable de celle de la "purple layer» (couche pourpre) et pourrait correspondre à une contamination par le niveau archéologique.

Le relevé préliminaire du bloc $\mathrm{K}$ a été réalisé à l'aide de la technique du relevé sur photographie (Lorblanchet 1995; Fritz et Tosello 2007 ) (cf. fig. 5). Cette méthode répond en tous points aux exigences de conservation des surfaces ornées et présente une grande fidélité à l'original. 
La technique se décompose en quatre étapes :

1. une couverture en photographie numérique haute résolution est réalisée (en respectant les contraintes d'orthogonalité et de distance) avec des éclairages d'incidences variées (lumière du jour et éclairage artificiel) ;

2. les images sont ensuite traitées en infographie afin d'en améliorer la lisibilité ou le contraste. Une mosaïque du bloc a ainsi été réalisée à partir de 10 clichés ;

3. face à l'original, le décalque sur film transparent des données visibles sur les photographies est effectué ;

4. l'ultime étape concerne la mise au net des relevés qui sont scannés et dont le rendu est traité par infographie.

Depuis cette première étude, réalisée en 2007, nous avons choisi d'appliquer deux nouvelles méthodes de relevé, l'une ayant recours à la prise de points topographiques couplés a l'enregistrement Gigapan ${ }^{3}$ et l'autre en utilisant un scanner $3 \mathrm{D}^{4}$ à courte portée (Minolta VI 9105). Chacune de ces méthodes vise à améliorer la réalisation du relevé et la lecture des traces de façonnage, mais également à faciliter la manipulation, virtuelle, de ces supports volumineux et par là même, leur conservation. Elles ont, par ailleurs, l'avantage de contribuer à la documentation en haute résolution du bloc afin de suivre l'évolution et les états de surface du document.

Figure 5 - Bloc K. Mosaïque photographique ; relevé graphique effectué sur le support 3D (pour les fragments 1 à 7 ) avec remontage de l'ensemble des fragments; numérotation des fragments et test de remontage des fragments 1 à 7 par numérisation 3D.

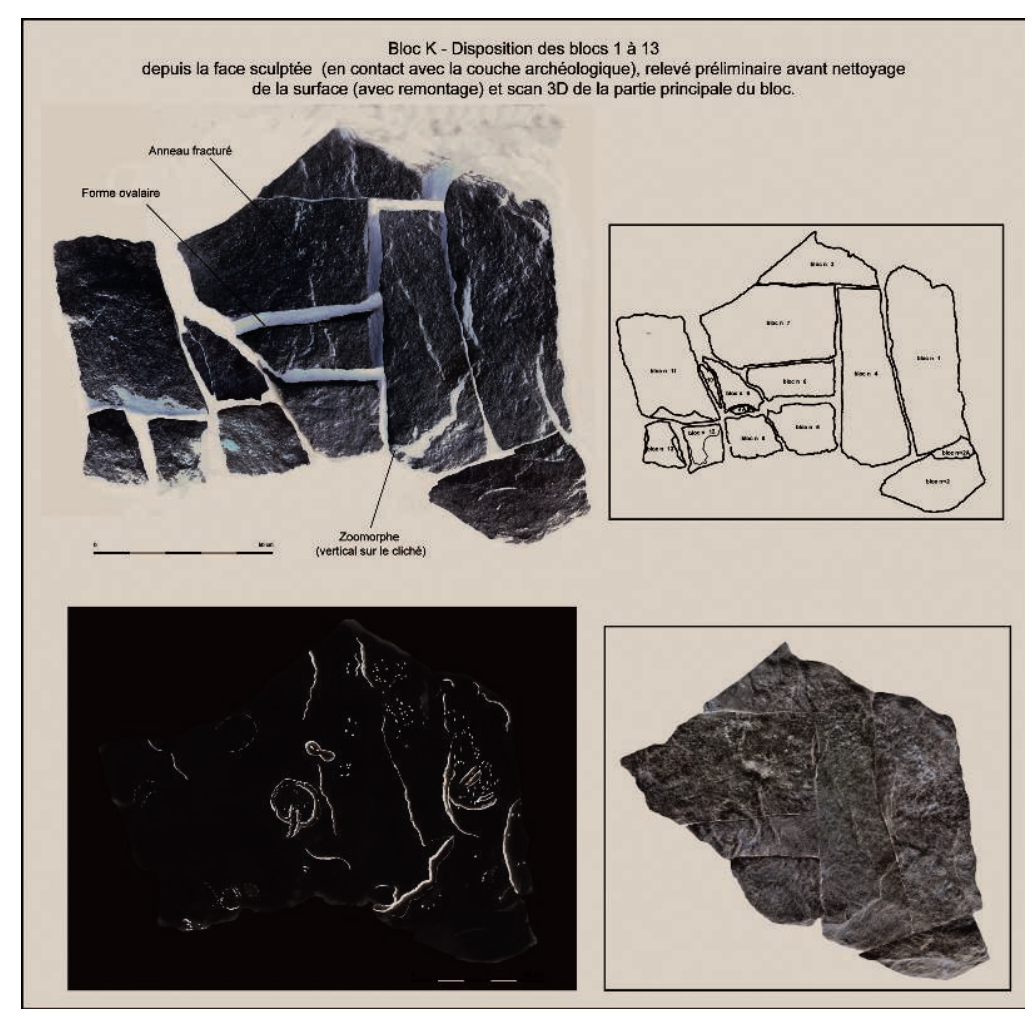

\section{Entités graphiques visibles et techniques de réalisation}

L'intégralité de la surface ornée du bloc semble avoir été travaillée, comme le suggère la présence de traces techniques (martelage, piquetage - Beaune (de) 1993 ; Delluc et 
Delluc 1978), mais cela reste à vérifier après nettoyage (cf. fig. 5). Malgré la présence de sédiments sur la surface ainsi qu'une fine pellicule carbonatée gênant la lecture des vestiges, trois éléments graphiques sont reconnaissables : une figure zoomorphe, une forme ovalaire, deux cupules larges.

L'alternance de zones fortement creusées et de zones en relief est particulièrement bien lisible sur la surface du bloc K. L'une des extrémités est ainsi fortement creusée, alors qu'au centre du bloc, un tracé zoomorphe a été réalisé en bas-relief et qu'à l'autre extrémité on relève, un second tracé en bas-relief accentuant ces différences de niveau.

La figure zoomorphe est traitée en léger bas-relief dans sa partie distale et en tracé piqueté profond dans sa partie mésiale (cf. fig. 5). Le statut façonné de la partie en basrelief reste à confirmer. Cette figure se compose d'un avant-train présentant un chignon en relief, d'une patte avant pointue et de la ligne de ventre. Le reste de la figure est difficilement déchiffrable et n'a peut-être jamais été représenté. Il pourrait vraisemblablement s'agir de la figuration d'un bovidé au contour schématique.

Deux cupules alignées avec des traces très nettes de piquetage se situent dans la partie supérieure du fragment (cf. fig. 5). Ces deux cupules peuvent être en relation avec la présence d'un anneau. Même si l'absence de fractures du pont jette un doute sur cette première interprétation. De plus, la présence de piquetage (dont la forme de l'impact suggère qu'il s'agit d'une percussion perpendiculaire) entre les deux cupules ne s'observe pas sur les anneaux aurignaciens en général. Cependant, il pourrait s'agir là d'un anneau cassé dont les pans de fractures et le fond ont été retravaillés par la suite.

Enfin, la troisième figure est un signe ovalaire dont les contours sont particulièrement travaillés, larges (entre 1 et $2 \mathrm{~cm}$ ) et profonds (environ $0,5 \mathrm{~cm}$ ) (fig. 6). Il s'agit de la première entité identifiée sur le bloc lors de son extraction. Réalisée en piquetage, elle propose également une phase de régularisation du fond de trait. À sa base, le tracé est échancré ou «invaginé » si l'on utilise les termes de B. et G. Delluc (Delluc et Delluc 1978). Son interprétation est encore sujette à discussion. La plus évidente, en comparaison des autres motifs de ce type connus pour la période aurignacienne, reste celle d'un sexe féminin ovalaire. La difficulté de lecture réside dans la figuration d'un tracé courbe fortement mis en relief à sa base. Ce dernier pourrait être l'illustration d'une fente vulvaire mais en volume, ce qui jette un doute sur cette interprétation. Une dernière proposition, plus discutable, serait celle de l'illustration d'un sexe masculin, thème connu pour ces phases anciennes de l'Aurignacien (Delluc et Delluc 1978; Bourrillon 2009 ; Bourrillon et al. 2012).

En ce qui concerne les techniques employées, elles sont "classiques" pour cette époque aurignacienne dans cette région. Des traces de martelage reconnaissables sur une grande partie de la surface du bloc suggèrent une mise en forme préalable à la gravure (cf. fig. 5). Les entités graphiques sont réalisées par piquetage avec, pour certains tracés, une régularisation par raclage (à confirmer). Cela se traduit par un tracé plus ou moins irrégulier avec des vestiges de cupules sur ses bords (cf. fig. 6).

Un autre point technique est observable: la mise en relief d'une partie de la surface rocheuse. Il s'agirait plus exactement d'un relief par réserve ${ }^{6}$ comme le suggèrent les traces de façonnage dans la partie périphérique de l'avant-train du zoomorphe.

De façon à comprendre avec davantage de précision la chaîne opératoire de réalisation des entités graphiques sur supports rocheux et les techniques utilisées, une expérimentation sur support calcaire est programmée. De plus, cette dernière 
permettra d'aborder la question de la relation fonctionnelle entre les œuvres pariétales et les vestiges matériels (outils et déchets), ayant pu participer à leur réalisation.

Les thématiques et les techniques identifiées ici s'intègrent parfaitement à celles déjà reconnues pour cette période aurignacienne dans la région du Périgord (Delluc et Delluc 1978; Bourrillon et White 2007 ; White et al. 2012). Mais le plus important réside dans la découverte de ce fragment de voûte ornée en contexte qui conduit à réviser non seulement la lecture (graphique et technologique) des blocs ornés mais aussi à réfléchir sur leur répartition dans le site au travers des archives disponibles. Les similitudes entre les sites de Castanet et Blanchard amènent à les intégrer conjointement dans cette nouvelle recherche. Il apparaît en effet essentiel de préciser le contexte de ces blocs ainsi que le moment de leur ornementation (en paroi ou au sol) pour comprendre la place occupée par ces pratiques. Ces recherches sont en cours et, aujourd'hui, engagées dans le cadre d'une Bourse de la Fondation Fyssen.

Figure 6 - Bloc K, forme ovalaire et détail des traces de piquetage.

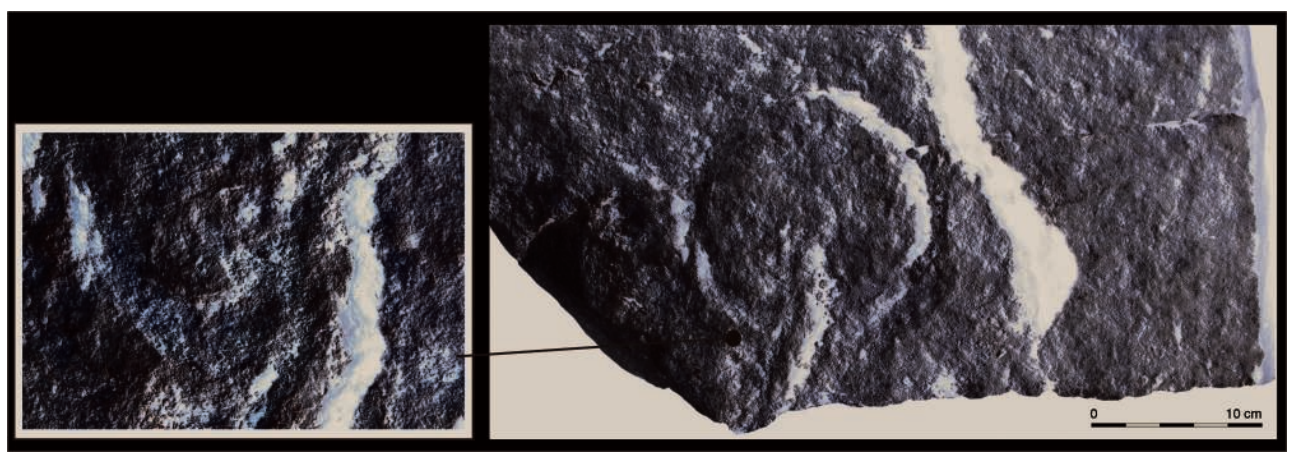

\section{4 - Travaux de contextualisation}

\section{La couche archéologique}

Le prélèvement maitrisé du bloc $\mathrm{K}$ a permis de conserver la couche archéologique intacte et d'en observer la morphologie et la composition.

Les fragments du bloc ont laissé de manière très nette leur empreinte sur le niveau archéologique : sur le sédiment, les négatifs d'au moins deux des gravures repérées lors du relevé ont été observés. Sous le bloc, le niveau présente des variations colorimétriques et texturales: une surface de coloration jaune dominée par des plaquettes calcaires avec du matériel fragmenté à son contact ainsi qu'un lambeau de couche pourpre («purple layer ») sur l'angle nord-ouest ont pu être distingués. Il s'agit de la couche A fouillée par D. Peyrony et M. Castanet, qui devait s'étendre au nord de la coupe où elle était extrêmement riche en matériel archéologique. Les plaquettes correspondent à une remontée du socle rocheux qui livre ici des éléments de modules plus petits que dans la partie sud de l'abri. Comme nous l'avons vu, le niveau archéologique du secteur nord concerne l'occupation de la partie avant de l'abri, le bloc $\mathrm{K}$ reposant sur un seuil marqué par une remontée du substrat. 


\section{L'attribution culturelle}

38 Le secteur nord et sa couche pourpre a livré une grande quantité de mobilier clairement attribuable à l'Aurignacien ancien lors de l'opération de diagnostic de 1994. La surface restreinte fouillée sous le bloc a livré peu de matériel mais est également caractéristique de l'Aurignacien ancien, avec deux perles en « forme de panier » (dont une ébauche), une baguette brute en bois de renne obtenue par fracturation, quelques lames retouchées $(n=4)$, une lame aurignacienne, des grattoirs sur lame $(n=3)$ et un burin d'angle (cf. fig. 2). Même si nous ne pouvons être affirmatifs sur la correspondance stricte entre les secteurs nord et sud, ces deux ensembles ne sont pas distinguables culturellement .

\section{La datation}

Un fragment d'os collé à la surface ornée du bloc K et cinq autres, prélevés dans la couche archéologique sous-jacente, ont été datés par la Oxford Radiocarbon Accelerator Unit, après traitement par ultrafiltration moléculaire (White et al. 2012). Parfaitement cohérentes entre elles, ces dates donnent un âge moyen non-calibré de $32400 \mathrm{BP}$, avec des fourchettes d'erreur très resserrées. Les déterminations ${ }^{14} \mathrm{C}$ des secteurs sud et nord du site ne sont pas statistiquement discernables les unes des autres, y compris celles du secteur sud faites par le laboratoire de Gif-sur-Yvette (tabl. 1).

La modélisation «Bayesienne » a permis, à l'aide de OxCal 4.1 (Bronk Ramsey 2001, 2009a, 2009b) et de INTCAL09 (Reimer et al. 2009) qui permettent de générer des courbes d'étalonnage, d'évaluer la chronologie du secteur nord. Ces analyses montrent que les premiers moments de l'occupation du site de Castanet sont datés entre 37 190-36 630 BP ( $68,2 \%$ prob.) et $37880-36530$ BP (95,4\% prob.). La fin de cette occupation est quant à elle comprise entre $36760-36330$ BP (68,2\% prob.) et 37 000-35 $770 \mathrm{BP}(95,4 \%$ prob.). Ainsi, ces résultats révèlent une période d'activité relativement brève, entre 36 940-36 $510 \mathrm{BP}(68,2 \%$ prob.).

41 Nous pouvons considérer que ces données radiométriques donnent une estimation assez précise du niveau archéologique "purple layer ». L'absence de dépôts entre le niveau aurignacien et la surface inférieure du bloc $\mathrm{K}$ nous amène à penser que l'hypothèse la plus vraisemblable est que celui-ci est tombé sur un sol d'occupation actif ou récemment abandonné.

42 Si nous ne sommes pas en mesure de déterminer le temps qui s'est écoulé entre la constitution du niveau archéologique et son scellement par le bloc, nous considérons à l'heure actuelle ces dates comme le terminus ante quem de l'ornementation du bloc. Précisons qu'une datation de $24950 \pm 240$ a été obtenue sur un fragment d'os brûlé situé au-dessus de la voûte effondrée, indiquant une faible présence gravettienne suite à la destruction de l'abri. Afin de confirmer cette hypothèse, une étude de faisabilité OSL sous le prolongement du bloc $\mathrm{K}$ encore présent dans la coupe a été engagée pour dater l'effondrement de la voûte.

43 La surface ornée du bloc K est donc relativement ancienne, même pour un art pariétal aurignacien dont la grotte Chauvet fournit la majeure partie des données radiométriques absolues. La comparaison de l'ensemble des datations de Castanet avec celles des prélèvements pariétaux de la grotte Chauvet (White et al. 2012) suggère que 
les images de Castanet sont légèrement plus anciennes, si l'on admet l'hypothèse de leur synchronie avec le niveau d'occupation du site.

Tableau 1 - Datations 14C du secteur nord et sud de l'abri Castanet (d'après White et al. 2011).

\begin{tabular}{|c|c|c|c|c|c|}
\hline OXA & Echantillon & Unité Strati. & Espèce & Date & $4 \%$ \\
\hline 21558 & Sud G11A 177 & 114 & Renne & 32350 & 450 \\
\hline 21559 & Sud G11A 179 & 114 & Renne & 33250 & 500 \\
\hline 21560 & Sud G11A 180 & 114 & Renne & 32800 & 450 \\
\hline 21561 & Sud G12A 242 & 110 & Os de mammifère (ind.) & 32050 & 450 \\
\hline 21562 & Sud G12A 244 & 110 & Renne & 32550 & 450 \\
\hline 21563 & Sud G12C 122 & 110 & Renne & 32600 & 450 \\
\hline 21564 & Sud G12C 129 & 110 & Renne & 32950 & 500 \\
\hline 21566 & Sud G12A 252 & 110 & Herbivore de taille moyenne & 32550 & 600 \\
\hline 21639 & Nord-surface gravée & Purple layer & Os de mammifère (ind.) & 32900 & 500 \\
\hline 21640 & Nord SS6C-80 & Purple layer & Os de mammifère (ind.) & 31900 & 450 \\
\hline $21641^{\circ}$ & Nord SS6C-25 & Purple layer & Os de mammifere (ind.) & 31950 & 450 \\
\hline $21642^{\circ}$ & Nord SS6C-25 & Purple layer & Os de mammifère (ind.) & 32500 & 450 \\
\hline 21643 & Nord RR6B-1 & Purple layer & Os de mammifère (ind.) & 32200 & 450 \\
\hline 21644 & Nord RR5C-8 & Purple layer & Os de mammifere (ind.) & 32350 & 450 \\
\hline 21645 & Nord J13C-22 & Purple layer. & Os de mammifere (ind.) & 32000 & 450 \\
\hline Gif & Échantillon & Unité Strati. & Espèce & Date & $4 /-$ \\
\hline 97313 & Sud I11 A 243 & 131 & Os de mammifère (ind.) & 32750 & 460 \\
\hline 97312 & Sud 111 A 290 & 131 & Os de mammifère (ind.) & 32460 & 420 \\
\hline 99166 & Sud H12 D 131 & 131 & Os de mammifère (ind.) & 34320 & 520 \\
\hline 99165 & Sud I12D 158 & 114 & Os de mammifère (ind.) & 31430 & 390 \\
\hline 99179 & Sud H12D 138 & 122 & Os de mammifère (ind.) & 32310 & 520 \\
\hline 99180 & Sud H12 D 156 & 122 & Os de mammifère (ind.) & 32950 & 520 \\
\hline 97330 & Sud K13 A 1416 & 101 & Os de mammifère brûlé & 24950 & 240 \\
\hline
\end{tabular}

\section{L'analyse du matériel archéologique comme élément de contextualisation du bloc}

\section{Le matériel en relation physique avec le bloc orné}

44 L'intérêt majeur de l'étude du mobilier est de fournir de précieux éléments sur le contexte du bloc orné. Il nous est en effet possible, grâce à l'examen des vestiges, d'affirmer que ce matériel a subi un choc conséquent, lequel correspond très probablement à la chute du bloc. L'étude du matériel archéologique permet également de disposer de quelques arguments d'ordre taphonomique en faveur de la chute in situ du bloc et de très faibles déplacements post-dépositionnels, tant pour le bloc que pour les vestiges (Chiotti et Cretin 2007 ; Cretin et Chiotti 2008). Les arguments que nous pouvons avancer sont les suivants :

- le taux de fragmentation du matériel lithique est très important. Cette fracturation a pu être observée directement sur le terrain par la présence de pièces cassées sur place (fig. 7), parmi les 26 raccords effectués, 17 entrent dans cette catégorie. Certaines sont simplement fracturées, d'autres présentent des traces d'écrasements intenses et quelques-unes sont même recollées par un concrétionnement ultérieur. La fragmentation est également visible dans certains refus de tamis, qui présentent une grande majorité de petits cassons, morphologiquement distincts des habituels déchets de débitage (cf. fig. 7). 
- la proportion de débris, catégorie de vestiges lithiques tellement fracturés qu'aucune lecture technologique n'est possible (pas même l'orientation de la pièce) est également nettement plus élevée ( $17 \%$, contre $9 \%$ dans le secteur sud) ;

- des observations semblables ont été faites sur les vestiges osseux (faune et industrie), et des pièces visibles à la fouille n'ont pu être prélevées du fait de leur intense fragmentation. Cependant la multiplicité des facteurs de fragmentation (anthropiques, post-dépositionnels) de ce matériel n'autorise pas un parallèle strict avec les vestiges lithiques.

Enfin, grâce aux techniques de restitution spatiale mises au point par M. Sisk en collaboration avec A. Clark, la réflexion sur les interrelations entre le socle, la couche archéologique, les vestiges lithiques écrasés et les blocs (état de conservation compris) au moment de l'effondrement est engagée. Pour l'heure, la fenêtre d'observation semble encore trop réduite $\left(1,36 \mathrm{~m}^{2}\right.$ fouillés pour un volume inférieur à $\left.900 \mathrm{~cm} 3\right)$, mais nous espérons bien être en mesure d'observer les phénomènes d'écrasement des parties dures (vestiges lithiques et socle), d'amortissage et d'expulsion des parties plus élastiques (couche archéologique).

\section{Contextualisation géologique et géomorphologique}

Afin de repositionner le bloc $\mathrm{K}$ dans la ligne d'abri, une tentative de caractérisation des différents macro-faciès du calcaire constituant la falaise a été réalisée, ainsi qu'une étude géomorphologique du vallon dans le but de comprendre la mise en place et le fonctionnement des abris.

\section{Cadre géomorphologique}

Le massif dans lequel sont creusés les abris correspond au terme supérieur des calcaires du Coniacien (C4b in Guillot et al. 1979; Capdeville et Rigaud 1987 - c3Ez in Platel et al. 1999 ; Karnay et al.1999). Les faciès relevés ont de nombreux points communs avec les coupes décrites dans les alentours (notamment à La Roque Saint-Christophe, Platel et al. 1999). La succession permet de placer le calcaire au sein duquel l'abri s'est formé autour de la limite entre Coniacien moyen et Coniacien supérieur.

En de nombreux points du vallon, la structure des auvents n'est pas conservée. Seule subsiste la falaise abrupte sur laquelle il est souvent malaisé de distinguer les différents lithofaciès. En outre, les aménagements troglodytiques ont largement contribué à la disparition de figures identifiables sur les parois naturelles. Ce fait est à prendre en compte lors de l'étude de l'évolution naturelle de la voûte mais il revêt également une importance particulière lorsqu'il s'agit d'estimer le potentiel archéologique de tels secteurs, susceptibles d'avoir été fortement endommagés (Gardère et al. 2008).

\section{Caractérisation des lithofaciès de la falaise}

Les observations et relevés stratigraphiques des macro-faciès des falaises rive droite et rive gauche ont permis d'identifier, dans ce calcaire coniacien, quatre lithofaciès ( $\mathrm{A}, \mathrm{B}$, $C$ et $\mathrm{D})$ permettant d'éclairer l'histoire de la formation des lignes d'abris.

De bas en haut de la falaise on distingue :

- faciès A : calcaire tendre très bioturbé ; 
- faciès $B$ : constitué de deux strates aux limites particulièrement nettes présentant des ondulations régulières d'échelle métrique. La strate inférieure atteint 40 à $50 \mathrm{~cm}$ et la formation supérieure 35 à $40 \mathrm{~cm}$;

- faciès $C$ : ensemble plus ou moins bioturbé organisé en stratifications obliques visible sur environ $60 \mathrm{~cm}$;

- faciès D : calcaire bien cristallisé sans structure sédimentaire visible surmontant les trois autres faciès (seuls quelques joints de stratifications horizontaux sont visibles).

Figure 7 - Fragmentation de l'industrie lithique provoquée par l'effondrement de la voûte, observée sous le bloc $\mathrm{K}$. a : éclat fragmenté en place ; b : éclat fragmenté en place et cimentée a posteriori par un concrétionnement ; $\mathrm{:} \mathrm{lame} \mathrm{retouchée} \mathrm{fragmentée} \mathrm{sous} \mathrm{le} \mathrm{bloc} \mathrm{;} d$ : refus de tamis SS5C-36, trié par classes dimensionnelles, presque exclusivement constitué de cassons.

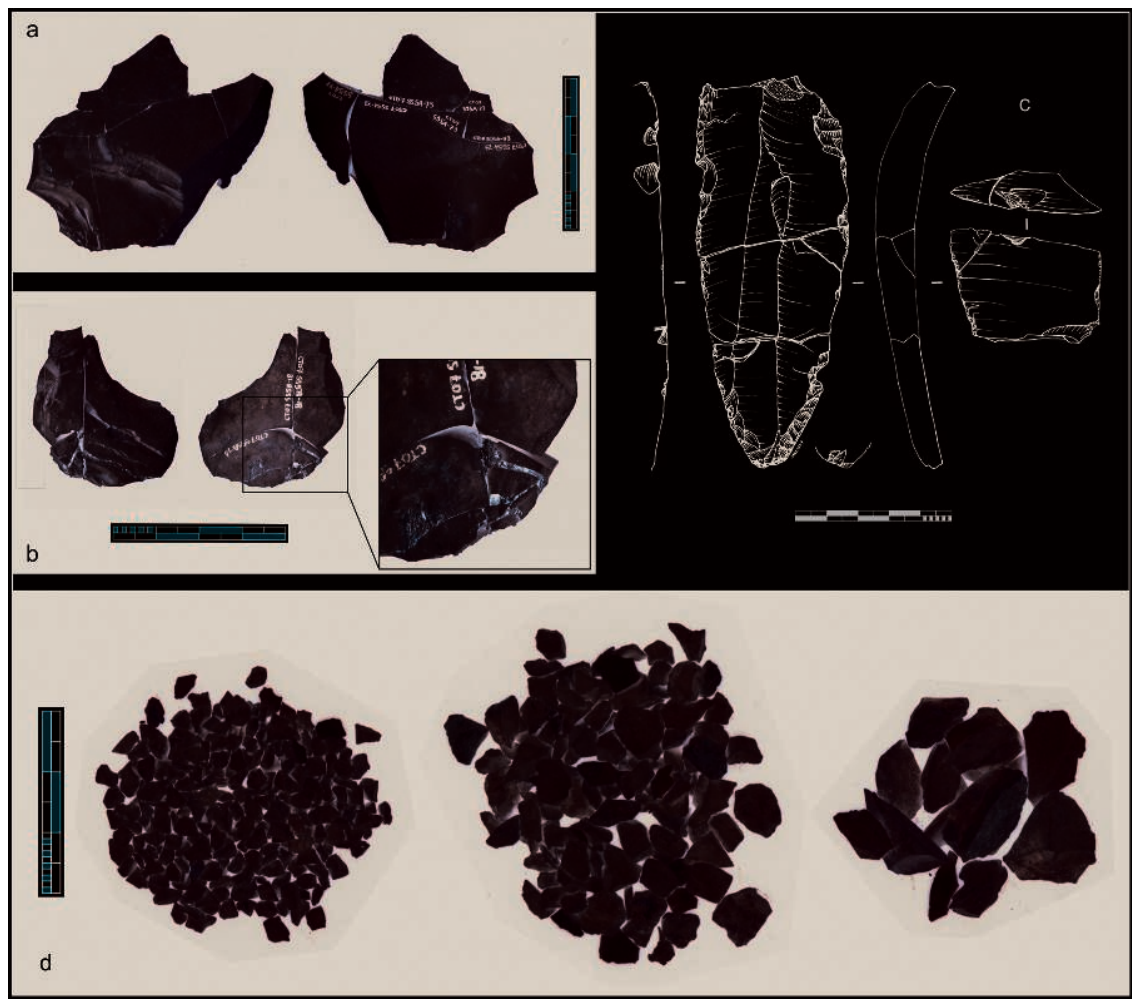

\section{Mise en place de la ligne d'abri}

La mise en place des abris résulte de la dégradation différentielle de la roche en fonction de la vulnérabilité intrinsèque des lithofaciès considérés. Ainsi, le faciès $\mathrm{A}$, très bioturbé, se dégrade rapidement sous l'effet des agents météorologiques. C'est dans cet horizon très vulnérable que se creusent les abris. Le faciès $\mathrm{B}$, constituant initial de la voûte, est également vulnérable à la cryoclastie. La bonne cohésion du calcaire assure le détachement de blocs de grande taille, tandis que les joints de stratifications délimitent la face supérieure du décollement. La combinaison des deux facteurs aboutit alors à un débitage $d u$ faciès $B$ en deux ensembles de grandes dalles planes et tabulaires. Le faciès $\mathrm{C}$, du fait de son hétérogénéité, est sujet à la dissolution. Le faciès $\mathrm{D}$ est le plus stable, il ne se débite que par arrachements gravitaires en blocs sans formes caractéristiques.

À la première logique horizontale de mise en place d'une ligne d'abris - par éboulisation du calcaire aux dépens des faciès les plus fragiles - vient se superposer une logique verticale induite par les cassures au sein du massif, telles que les diaclases ou 
les failles. Des figures de dissolution se retrouvent ainsi sur toute la hauteur de la falaise. À l'aplomb des cassures, la hauteur des auvents peut être fortement augmentée, $\mathrm{du}$ fait de la fragilisation de la voûte qui se détruit plus rapidement que dans les zones non faillées (structures en cloche).

\section{Un cas particulier : le secteur de la coupe Peyrony}

Figure 8 - Évolution de l'abri Castanet et formation des dépôts géoarchéologiques. Légende des photos de gauche à droite : $E$ = éboulis, $M=$ massif calcaire, $C=$ Castanet sud ; coupe Peyrony après extraction du bloc $K$, noté la présence de la continuité du bloc $K$ dans la coupe ; formation de l'éboulis post effondrement de la voute ; comblement intersticiel de l'éboulis par des formations carbonatés

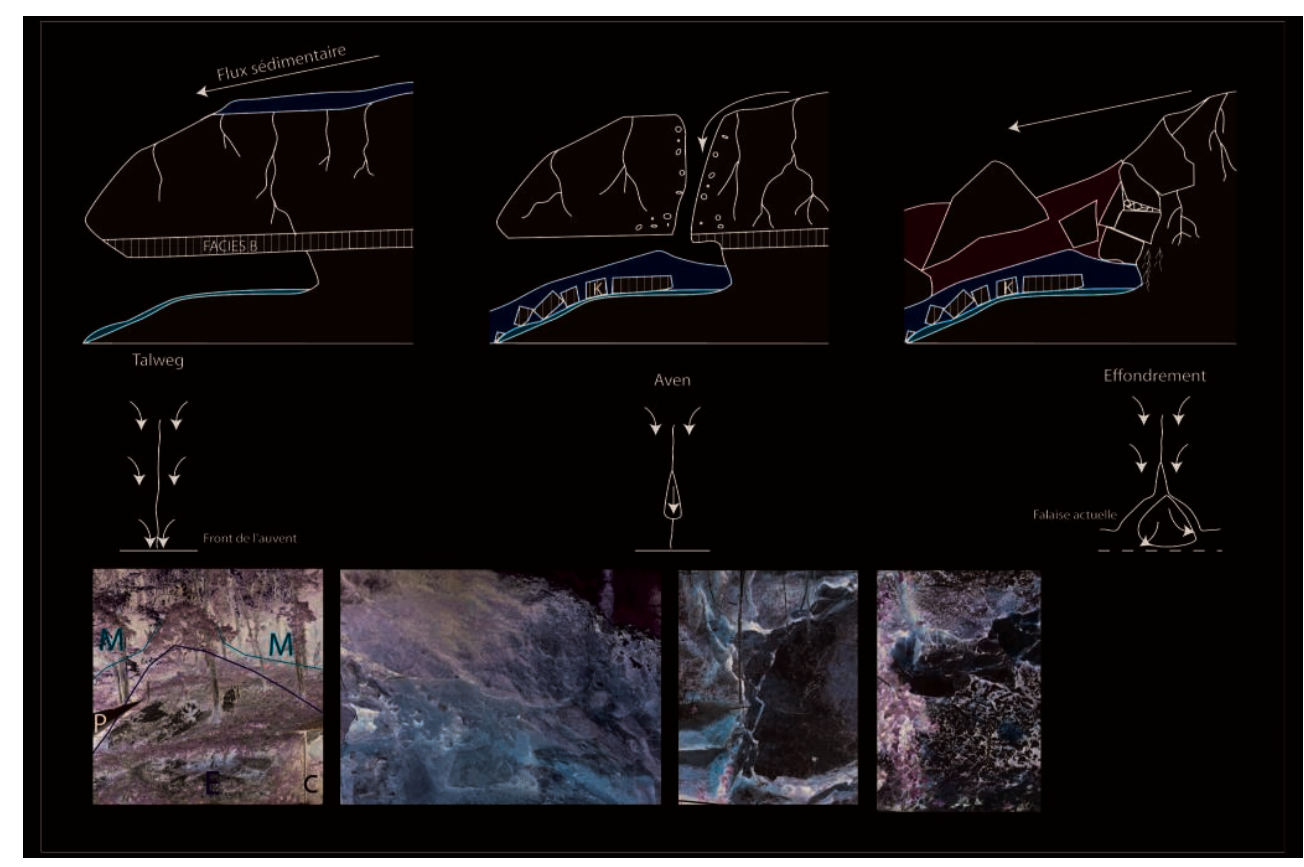

La coupe met en évidence une structuration en plusieurs étapes (fig. 8). Àla base, le niveau archéologique principal ("purple layer») est horizontal et repose directement sur le socle. Un premier épisode de destruction de l'auvent provoque la chute de dalles, issues du faciès $\mathrm{B}$, sur le matériel anthropique. C'est à ce moment que le bloc $\mathrm{K}$ se détache du massif calcaire. Sa taille, sa position dans la coupe et la trace qu'il a laissée dans le sédiment indiquent qu'il n'a pas été déplacé. L'absence de pendage de la couche sous-jacente et la très grande fréquence de vestiges lithiques concassés sur place éliminent également toute possibilité de glissement. Le bloc $\mathrm{K}$ est donc tombé à l'aplomb de sa position initiale sur la voûte. À la suite de cette première phase de déstructuration du secteur, la dynamique du comblement change: les apports postérieurs présentent un fort pendage vers l'ouest. La texture sédimentaire varie également, la blocaille est emballée dans une matrice argileuse, évoquant des formations ayant glissé depuis les topographies les plus hautes. Des indices probants d'alimentation à partir d'un aven aujourd'hui effondré ont été mis en évidence.

L'évolution du secteur de la coupe Peyrony met en lumière les différents mécanismes susceptibles d'affecter les cavités du vallon. Durant l'occupation par les Hommes, la morphologie de l'abri peut varier selon les modalités décrites précédemment. La chute des derniers éléments d'auvent marque le sommet des niveaux archéologiques. La 
déstabilisation du massif du fait des effondrements est accentuée par la dissolution induite par les zones fracturées. De très gros blocs peuvent alors glisser ou s'affaisser (par exemple au sud de l'abri Labattut sur le versant occidental ou dans le secteur de la coupe Peyrony), la paroi devient alors très abrupte.

\section{5 - Conclusion}

Les travaux qui viennent d'être présentés ont permis de préciser le processus d'évolution du site, depuis la formation de l'abri-sous-roche jusqu'à son occultation totale par un éboulis. À l'intérieur de celui-ci, il a été possible d'identifier les blocs d'effondrement de la voûte de l'abri et la place des occupations humaines dans ce dispositif.

Le contact entre les blocs effondrés de la voûte ornée et le niveau archéologique est direct comme en attestent en particulier les vestiges lithiques, ce qui nous a permis d'envisager un terminus ante quem pour les gravures de la voute. Afin de cerner mieux encore la chronologie relative de ces évènements, une étude de faisabilité de datations OSL est engagée. Celle-ci permettrait de dater la chute du bloc et le recouvrement par l'éboulis.

Quant au témoin d'art pariétal, découverte exceptionnelle, il s'agit d'un fragment de l'auvent, orné, qui protégeait l'occupation aurignacienne.

Les enjeux d'une poursuite de la fouille archéologique sont donc évidents : il existe, entre secteur nord et sud, plus de $7 \mathrm{~m}$ d'éboulis non fouillés qui permettraient d'agrandir la fenêtre d'observation et, peut-être, de découvrir d'autres éléments de l'auvent orné. Nous savons en effet qu'à l'intérieur de la coupe Peyrony, dans le prolongement $\mathrm{du}$ bloc $\mathrm{K}$, d'autres éléments rocheux sont à rattacher à ce plafond effondré.

59 Les axes de recherche, complémentaires visent à :

- mieux connaître le niveau d'occupation humaine, dans le but d'améliorer la représentativité de la surface fouillée, de déceler des zones d'activité différentes et, par conséquent, d'aborder la dimension palethnographique du gisement ;

- documenter le contexte géomorphologique et trouver le prolongement de la voûte, peutêtre orné ;

- établir une relation plus étroite encore entre les deux éléments précédents afin de parvenir à une meilleure compréhension du contexte social de l'installation et des activités humaines réalisées dans ce site exceptionnel.

Cet objectif ultime nécessite également l'extension de la réflexion et de la recherche à l'ensemble du vallon, aux autres éléments rocheux ornés et à leurs contextes. Le travail sur les archives et le mobilier découvert antérieurement (R. White et R. Bourrillon) permettent en effet déjà d'affirmer qu'il existe à la fois des décors sur les parois de l'abri et sur des blocs au sol, mais aussi que les sites de Castanet et de Blanchard présentent de nombreuses correspondances graphiques et techniques et ne sont peutêtre que deux parties d'une même occupation aurignacienne.

Cette recherche a été soutenue depuis 1994 par un certain nombre d'institutions que nous souhaitons remercier ici : la United States National Science Foundation, la Direction régional des affaires culturelles d'Aquitaine (DRAC-Aquitaine), the Partner University Fund (PUF), the Andrew Mellon Foundation, la L.S.B. Leakey Foundation, la Reed Foundation, la Rock Foundation, 
la Fine Foundation, l'UMI 3199-CNRS-NYU (Center for International Research in the Humanities and Social Sciences), l'Institute for Ice Age Studies, la Theodore Dubin Foundation, le Service archéologique départemental (SAD) de la Dordogne, la New York University, la Fondation Fyssen et la Fulbright Foundation.

Les auteurs tiennent également à remercier les personnes suivantes pour leur aide, leurs conseils et leur soutien : les familles Castanet et Castanet-Daumas, Hélène Talenton, Jean-Pierre Lagarde, Baptiste et Edith Burg, John Yellen, Dany Barraud, Nathalie Fourment, Jean-Jacques Cleyet-

Merle, Serge Maury, Jean-Michel Geneste, Emilienne Baneth-Nouailhetas, Christophe Goddard, Edward Berenson, Mike Habermann, Lois Dubin, Jean-Christophe Portais, Philippe Jugie, Jacqueline Angot-Westin, Eric Capdeville, Christophe Vigerie, Jean et Annie Labeille, Jean-Paul Blémont, Philippe Mourneau.

\section{BIBLIOGRAPHIE}

AZÉMA M., GÉLY B., BOURRILLON R., LHOMME D. 2012 - La grotte ornée paléolithique de Baume Latrone (France, Gard) : la 3D remonte le temps... In : J. Clottes (dir.), l'Art pléistocène dans le monde. Congrés IFRAO, Tarascon-sur-Ariège, septembre 2010, Symposium « Application des techniques forensiques aux recherches sur l'art pléistocène ». Préhistoire, Art et Sociétés, LXV-LXVI, p. 214-215 et CD p. 1222-1238.

BEAUNE (de) S. 1993 - Approche expérimentale de techniques paléolithiques de façonnage de roches peu aptes à la taille. Paleo, 5, p. 155-177.

BON F. 2002 - L'Aurignacien entre Mer et Océan. Réflexion sur l'unité des phases anciennes de l'Aurignacien dans le Sud de la France. Joué-Lès-Tours : Société Préhistorique Française, mémoire XIX, $253 \mathrm{p}$.

BOURRILLON R. 2007 - Analyses et relevés préliminaires. In : R. White (Ed.), Abri Castanet, secteurs Sud et Nord (Commune de Sergeac, Dordogne). Rapport de Fouille Programmée, années 2006-2008. Bordeaux : Service Régional de l'Archéologie, Ministère de la Culture, p. 109-120.

BOURRILLON R. 2009 - Les représentations humaines sexuées dans l'art du Paléolithique supérieur européen : diversité, réminiscences et permanences. Toulouse : Université de Toulouse II le Mirail, 2009, 2 t., 552 p., ill, Thèse de 3e cycle : Archéologie : Toulouse II.

BOURRILLON R., FRITZ C., SAUVET G. 2012 - La thématique féminine au cours du Paléolithique supérieur européen : permanences et variations formelles. Bulletin de la SPF, 109, nº 1, p. 85-103.

BOURRILLON R., WHITE R. 2007 - Le bloc K de Castanet dans l'Aurignacien d'Aquitaine In : R. White (Ed.), Abri Castanet, secteurs Sud et Nord (Commune de Sergeac, Dordogne). Rapport de Fouille Programmée, années 2006-2008. Bordeaux : Service Régional de l'Archéologie, Ministère de la Culture, p. 121-124.

BOURDIER C., FUENTES O., HAMON G., PINÇON G. 2008 - Technologies 3D appliquées à la sculpture pariétale magdalénienne. In : O. Buchsenchutz (Éd.), Images et relevés archéologiques, de la preuve à la démonstration. Actes du 132e Congrès national des Sociétés Savantes Historiques et Scientifiques (2007). Arles, CDRom. 
BRONK RAMSEY C. 2001 - Development of the radiocarbon calibration program OxCal. Radiocarbon, 43, n² A, p. 355-363.

BRONK RAMSEY C. 2009a - Bayesian analysis of radiocarbon dates. Radiocarbon, 51, $\mathrm{n}^{\circ} 1$, p. 337-360.

BRONK RAMSEY C. 2009b - Dealing with outliers and offsets in radiocarbon dating. Radiocarbon, 51, n’ 3, p. 1023-1045.

CAPDEVILLE J.-P., RIGAUD J.-Ph. 1987 - Carte géologique 1/50 000, Notice explicative, feuille Sarlat - La Canéda. Orléans : 808, BRGM.

CHIOTTI L. 2005 - Les Industries lithiques aurignaciennes de l'abri Pataud, Dordogne, France : les fouilles de Hallam L. Movius Jr., Oxford, BAR International Series, Archaeopress, 839 p.

CHIOTTI L., CRETIN C. 2007 - Étude du matériel lithique de l'abri Castanet (secteur sud et coupe Peyrony) Campagnes 2005-2007. In : R. White (Éd.), Abri Castanet, Nouveau Secteur. Rapport de Fouille Programmée, année 2007. Bordeaux : Service Régional de l'Archéologie, Ministère de la Culture, p. 132-155.

CRETIN C., CHIOTTI L. 2008 - Le matériel lithique de l'abri Castanet. Bilan des campagnes 2005-2008. In : R. White (Ed.), Abri Castanet, Nouveau Secteur. Rapport de Fouille Programmée, années 2006-2008. Bordeaux : Service Régional de l'Archéologie, Ministère de la Culture, p. 144-195.

DELLUC B. et G. 1978 - Les manifestations graphiques aurignaciennes sur support rocheux des environs des Eyzies (Dordogne). Paris : Gallia Préhistoire, CNRS, 21, nº 1 et 2, p. 213-438, 96 fig.

DELPORTE H. 1984 - Le grand abri de La Ferrassie. Fouilles 1968-1973. Publications de l'Université de Provence : Études Quaternaires, mémoire ${ }^{\circ}$ 7, $277 \mathrm{p}$.

CHAMPAGNE F., ESPITALIÉ R. 1981 - Le Piage, site préhistorique du Lot. Paris : Mémoires de la Société Préhistorique Française, XV, 208 p.

FRITZ C., TOSELLO G. 2007 - The Hidden Meaning of Forms : Methods of Recording Paleolithic Parietal Art. New-York : Journal of Archaeological Method and Theory, 14, n 1, p. 48-80.

FRITZ C., TOSELLO G., PÉRAZIO G., PÉRAL J., GUICHARD L. 2010 - Technologie 3D et relevé d'art pariétal : une application inédite dans la grotte de Marsoulas. 臨En Ligne帛,

http://www.revue.inventaire.culture.gouv.fr/insitu/insitu/, Ministère de la Culture.

GARDÈRE P., WHITE R., SISK M., CLARK A. 2008 - Un travail en cours : les abris Castanet et Blanchard dans leur contexte géomorphologique, géokarstique et paléotopographique. In : R. White (Ed.), Abri Castanet, Nouveau Secteur. Rapport de Fouille Programmée, année 2008. Bordeaux : Service Régional de l'Archéologie, Ministère de la Culture, p. 92-143.

GUILLOT P. L., FEYS R., LEFAVRAIS-RAYMOND A., PLATEL J. P., LEFAVRAIS-HENRY M., BASSOULET J. P., CONTINI D., GOURDON-PLATEL N., QUARANTOTTI G., VANACKER J. C., TOILLIEZ L., PARFENOFF A. 1979 - Carte géologique 1/50 000, Notice explicative, feuille de Terrasson, 784. Orléans : BRGM.

KARNAY G., avec la collaboration de AUJOULAT N., KONIK S., MAUROUX B., PLUCHERY E., TURQ A. 1999 -Carte géologique 1/50 000, feuille Le Bugue (807), Notice explicative. Orléans : BRGM, 91 p.

LORBLANCHET M. 1995 - Les grottes ornées de la Préhistoire -Nouveaux regards. Paris : Errance, 288 p.

MENSAN R. 2007 - Poursuite de la fouille de l'abri Castanet. In : R. White (Ed.), Abri Castanet, Nouveau Secteur. Rapport de Fouille Programmée, année 2007. Bordeaux : Service Régional de l'Archéologie, Ministère de la Culture, p. 60-68. 
MENSAN R., SISK M. 2008 - Poursuite de la fouille de l'abri Castanet : 2007 et 2008, In : R. White (Ed.), Abri Castanet, Nouveau Secteur. Rapport de Fouille Programmée, année 2006-2008. Bordeaux : Service Régional de l'Archéologie, Ministère de la Culture, p. 64-77.

MOUTHON A., JOFFROY R. 1958 - Le gisement aurignacien des Rois à Mouthiers (Charente). Paris : Gallia-Préhistoire, IXème supplément, CNRS, 140 p.

PELEGRIN J., WHITE R. 1998 - Abri Castanet : Rapport de Fouille Programmée, années 1996-1998. Bordeaux : Service Régional de l'Archéologie, Ministère de la Culture.

PEYRONY D. 1935 - Le gisement Castanet, Vallon de Castelmerle, commune de Sergeac (Dordogne), Aurignacien I et II. Bulletin de la Société Préhistorique Française, 32, p. 418-443.

PINÇON G. 2004 - Méthodes de relevé appliquées à l'étude de l'art pariétal sculpté magdalénien du Roc-aux-Sorciers (Angles-sur-l'Anglin, France). Bulletin de l'Association des Archéologues de Poitou-Charentes, 33, p. 41-58.

PLATEL J. P., avec la collaboration de FAUGERAS P., MAUROUX B., SPENCER C., CHARNET F, CÉLERIER G., HARIELLE B., JACQUEMENT P. 1999 - Carte géologique 1/50 000, feuille Thenon (783), Notice explicative. Orléans : BRGM, $129 \mathrm{p}$.

REIMER P.J., BAILLIE M.G.L., BARD E., BAYLISS A., BECK J.W., BLACKWELL P.G., BRONK RAMSEY C., BUCK C.E., BURR G., EDWARDS R.L., FRIEDRICH M., GROOTES P.M., GUILDERSON T.P., HAJDAS I., HEATON T.J., HOGG A.G., HUGHEN K.A., KAISER K.F., KROMER B., MCCORMAC F.G., MANNING S.W., REIMER R.W., RICHARDS D.A., SOUTHON J.R., TALAMO S., TURNEY C.S.M., VAN DER PLICHT J., WEYHENMEYER C.E. 2009 - Intcal09 and Marine09 radiocarbon age calibration curves, 0-50,000 years cal BP. Radiocarbon, 51, $\mathrm{n}^{\circ}$ 4, p. 1111-1150.

RIGAUD J. Ph. 1993 - L'Aurignacien dans le Sud-Ouest de la France, bilan et perspectives. In : L. Banesz et J. Kozlowski (Ed.), Aurignacien en Europe et au Proche-Orient.Actes du colloque organisé par la Commission VIIIe de l'UISPP à Bratislava (1-7 septembre1991). Nitra-Bratislava : Institut Archéologique de l'Académie Slovaque des Sciences, p. 181-186 (vol. 2).

SANOIT (de) J., CHAMBELLAN D., PLASSARD F. 2005 - Caractérisation in situ du pigment noir de quelques œuvres pariétales de la Grotte de Rouffignac à l'aide d'un système portable d'analyse par fluorescence X (XRF). ArchéoSciences, 29, p. 61-68.

SISK M. L. 2010 - Three-Dimensional Gigapan Views of Archaeological Sites and Artifacts : Examples from the Paleolithic of Southwest France. In : Proceedings of the Fine International Conference on Gigapixel Imaging for Science, (November 11-13 2010), Pré-Actes, En ligne,URL : http://gigapixelscience.gigapan.org/papers-2?offset=30.

SONNEVILLE-BORDES (de) D. 1960 - Le Paléolithique supérieur en Périgord. Bordeaux : Delmas, 558 p.

TARTAR É. 2007 - L'industrie en os et en bois de renne. In : White R. (Ed), Abri Castanet, Nouveau Secteur. Rapport de Fouille Programmée, année 2007. Bordeaux : Service Régional de l'Archéologie, Ministère de la Culture, p. 156-164.

TARTAR É. 2009 - De l'Os à l'Outil : Caractérisation technique, économique et sociale de l'utilisation de l'os à l'Aurignacien ancien. Étude de trois sites : l'Abri Castanet (secteurs nord et surd), Brassempouy (Grotte des Hyènes et abri Dubalen) et Gatzarria. Paris : Université de Paris I-Panthéon-Sorbonne, 2009, 2 t., $300 \mathrm{p}$. Thèse de $3^{\mathrm{e}}$ cycle : Archéologie : Paris I-Panthéon-Sorbonne.

TEXIER J. P. 2009 - Histoire géologique de sites préhistoriques classiques du Périgord : une vision actualisée : La Micoque, La grotte Vaufrey, Le Pech de l'Azé I et II, La Ferrassie, L'abri Castanet, Le Flageolet, Laugerie-Haute. Paris : CTHS, 193 p. 
TYMULA S. 1999 - L'art du Roc-de-Sers (Charente) dans son contexte solutréen. Paris : Université de Paris I (Panthéon-Sorbonne), 1999. 2 t., 845 p., ill. Thèse ND : Sc. : Paris I.

WHITE R. 2005 - Abri Castanet, Nouveau Secteur. Rapport de Fouille Programmée, année 2005. Bordeaux : Service Régional de l'Archéologie, Ministère de la Culture, 110 p.

WHITE R. 2006 - Abri Castanet, Nouveau Secteur. Rapport de Fouille Programmée, année 2006. Bordeaux : Service Régional de l'Archéologie, Ministère de la Culture, 184 p.

WHITE R. 2007a - Abri Castanet, Nouveau Secteur. Rapport de Fouille Programmée, année 2007. Bordeaux : Service Régional de l'Archéologie, Ministère de la Culture, 186 p.

WHITE R. 2007b - Morphologie de l'abri et étude du réseau karstique sous Castel-Merle. In : White R. (Éd.), Abri Castanet, Nouveau Secteur. Rapport de Fouille Programmée, année 2007. Bordeaux : Service Régional de l'Archéologie, Ministère de la Culture, p. 69-73.

WHITE R. 2007c - Sergeac. L'abri Castanet.Bilan scientifique de la région Aquitaine, 2005. Bordeaux : Service régional de l'Archéologie, Drac Aquitaine, Ministère de la Culture et de la Communication, p. 52.

WHITE R. 2008a - Abri Castanet, Nouveau Secteur. Rapport de Fouille Programmée, années 2006-2008. Bordeaux : Service Régional de l'Archéologie, Ministère de la Culture, 292 p.

WHITE R. 2008b - Objets de parure et objets osseux décorés à l'abri Castanet, Secteurs sud et nord 2005 à 2008. In : White R. (Éd.), Abri Castanet, Nouveau Secteur. Rapport de Fouille Programmée, années 2006-2008. Bordeaux : Service Régional de l'Archéologie, Ministère de la Culture, p. 244-274.

WHITE R. 2008c - Sergeac. Abri Castanet.Bilan scientifique de la région Aquitaine, 2006. Bordeaux : Service régional de l'Archéologie, Drac Aquitaine, Ministère de la Culture et de la Communication, p. 42-44.

WHITE R. 2009a - Abri Castanet, Nouveau Secteur. Rapport de Fouille Programmée, année 2009. Bordeaux : Service Régional de l'Archéologie, Ministère de la Culture, 222 p.

WHITE R. 2009b - Sergeac. Abri Castanet. Bilan scientifique de la région Aquitaine, 2007. Bordeaux : Service régional de l'Archéologie, Drac Aquitaine, Ministère de la Culture et de la Communication, p. 60-61.

WHITE R., CRETIN C., CHIOTTI L., BOURRILLON R., SISK M., GARDERE Ph. 2007 - Fragment de la voûte gravée et colorée de l'abri Castanet (Secteur nord). In : White R. (Éd.), Abri Castanet, Nouveau Secteur. Rapport de Fouille Programmée, année 2007. Bordeaux : Service Régional de l'Archéologie, Ministère de la Culture, p. 74-131.

WHITE R., BOURRILLON R. 2008 - Avancement étude du bloc K. In : White R. (Ed.), Abri Castanet, Nouveau Secteur. Rapport de Fouille Programmée, années 2006-2008. Bordeaux : Service Régional de l'Archéologie, Ministère de la Culture, p. 81-91.

WHITE R., HIGHAM T. 2008 - Bilan des datations C14 pour le Secteur sud et nord. In : White R. (Ed.), Abri Castanet, Nouveau Secteur. Rapport de Fouille Programmée, années 2006-2008. Bordeaux : Service Régional de l'Archéologie, Ministère de la Culture, p. 78-80.

WHITE R., MENSAN R., SISK M., CLARK A. 2010 - Sergeac. Abri Castanet. Bilan scientifique de la région Aquitaine, 2008.Bordeaux : Service régional de l'Archéologie, Drac Aquitaine, Ministère de la Culture et de la Communication, p. 52-55.

WHITE R., MENSAN R., BOURRILLON R., CRETIN C., HIGHAM T., CLARK A., SISK M., TARTAR E., GARDERE P., PELEGRIN J., VALLADAS H., TISNERAT-LABORDE N., SANOIT (de) J., CHAMBELLAN D., CHIOTTI L. 2012 - Context and dating of a newly discovered Aurignacian « vulvar » 
representation from Abri Castanet, France. Proceedings of the National Academy of Science, 109, $\mathrm{n}^{\circ} 22$, p. 8450-8455.

\section{ANNEXES}

\section{Références pour les illustrations}

Si cette recherche est le résultat d'un travail commun de l'équipe Castanet, il est important de souligner la contribution particulière de certains membres de l'équipe et partenaires externes aux illustrations suivantes :

1 - C. Cretin et N. Maumont, Centre National de Préhistoire, fonds cartographique CMinistère de la Culture et de la Communication.

2 - É. Tartar, L. Chiotti, R. White, C. Cretin et R. Bourrillon. Photo du bloc gravé par Ph. Jugie OMNP Les Eyzies - Dist. RMN.

3 - P. Gardère, R. Mensan et P. Kervinio.

4 - R. White et R. Mensan.

5 - R. Bourrillon, C. Cretin et M. Sisk.

6 - R. Bourrillon.

Tabl. 1 - T. Higham, H. Valladas et N. Tisnerat.

7 - C. Cretin et L. Chiotti.

8 - Ph. Gardère.

\section{NOTES}

1. Voir à ce propos : Mouthon, Joffroy 1958 ; Champagne, Espitalié 1981 ; Delporte 1984 ; Rigaud 1993 ; Bon 2002 ; Chiotti 2005.

2. C'est ainsi que l'ordre de numérotation ne suit pas tout à fait la chronologie des évènements, puisqu'une inversion s'est glissée entre les blocs $\mathrm{K} 1$ et $\mathrm{K} 2$, celui-ci étant en réalité le premier enlevé. Par ailleurs, les fragments de bloc n'ayant pas emporté la surface ornée n'ont pas été numérotés. Ils sont désignés de cette façon: «fragment de bloc intermédiaire 3-4 » pour un élément détaché entre les blocs $\mathrm{n}^{\circ} 3$ et $\mathrm{n}^{\circ} 4$. Il y a en fait quatre blocs intermédiaires $(2-1,3-4,4-5$ et 4-5 bis).

3. Le Gigapan est un appareil robotique permettant de prendre une série (jusqu'à des centaines) d'images partiellement chevauchées et de les remonter en une seule image de haute résolution (0,5 - 2 gigapixels). Ensuite, un logiciel développé par l'équipe de GigaPan crée une mosaïque de l'ensemble des images. Le logiciel de GigaPan modifie la résolution de l'image en fonction du niveau de zoom, tenant compte de la navigation très rapide des images pesant fréquemment plus d'un gigabyte. Cet outil nous a offert l'occasion unique de documenter la totalité de la surface du bloc K dans une seule image (Sisk 2010). En plus d'aider à l'enregistrement, les images du GigaPan permettent de visualiser le bloc en trois dimensions (http://www.gigapan.org/viewProfile.php? userid=4885). La version la plus récente de cet appareil a été mise à notre disposition gratuitement par la Carnegie-Mellon University. 
L'application de cette technologie à l'art rupestre s'est fortement développée ces dernières années. Nous pouvons citer, par exemple, les travaux dans les abris du Roc-aux-Sorciers et de La Chaire-à-Calvin (Pinçon 2004 ; Bourdier et al. 2008), dans les grottes de la Baume Latrone (Azéma et al. 2012) et de Marsoulas (Fritz et al. 2010).

4. Le scanner Konica Minolta VI 910 a été gracieusement mis à disposition par le Laboratoire de Recherche TRACES UMR 5608, Toulouse.

5. «Le relief par réserve : ce procédé de taille directe consiste, pour obtenir un relief, à dégager le plan du fond tout autour des figures ou des motifs réservés. Le dégagement du plan du fond s'effectue par un tracé à section recticurviligne à un bord modelé et un bord abaissé, dont la profondeur peut atteindre $1 \mathrm{~cm}$ environ. Cette taille de réserve [...] confère au relief un aspect plat ou semi-méplat et peut être à fond de cuvette ou à fond plat » (Tymula 1999 - p. 232).

6. «Le relief par réserve : ce procédé de taille directe consiste, pour obtenir un relief, à dégager le plan du fond tout autour des figures ou des motifs réservés. Le dégagement du plan du fond s'effectue par un tracé à section recticurviligne à un bord modelé et un bord abaissé, dont la profondeur peut atteindre $1 \mathrm{~cm}$ environ. Cette taille de réserve [...] confère au relief un aspect plat ou semi-méplat et peut être à fond de cuvette ou à fond plat »(Tymula 1999 - p. 232).

\section{RÉSUMÉS}

En 2007, un fragment de voûte ornée a été découvert, sur le site de l'abri Castanet (commune de Sergeac, Dordogne) lors des opérations archéologiques ce qui n'était plus arrivé depuis 1912 (fouilles de M. Castanet pour L. Didon). La face profondément gravée et peinte de ce bloc d'effondrement, pesant plus d'une tonne, était en contact direct avec la couche archéologique aurignacienne. Une série de six datations par ${ }^{14} \mathrm{C}$ AMS (par filtration moléculaire), réalisée sur des vestiges osseux de faune en contact avec le sommet du niveau d'occupation, donne des résultats cohérents dont la moyenne est de 32400 BP. Parmi les tracés visibles, sur la surface du bloc, la figure la plus évidente est celle d'une vulve. Cette thématique a été mise en évidence de façon récurrente au cours des fouilles du $\mathrm{XX}^{\mathrm{e}}$ siècle sur le site de Castanet et sur celui voisin de Blanchard. La découverte de ce bloc et sa datation permettent de recadrer chronologiquement ceux retrouvés à quelques mètres au cours des fouilles anciennes et d'aborder ces manifestations du vallon de Castel-Merle sous un nouvel angle.

Here, we report on the discovery in 2007, in perfect archaeological context, of part of the engraved and ocre-stained undersurface of the rockshelter ceiling from Abri Castanet, Commune de Sergeac, Dordogne. The engraved/painted undersurface of the massive roof-collapse block, weighing more than a ton, was in direct contact with the surface of the early Aurignacian archaeological layer onto which it had fallen. A series of six molecular filtration dates on faunal bone from that surface are internally coherent and yield a mean age estimate of 32400 radiocarbon years BP. The clearest engraving observable on the newly discovered ceiling fragment fits morphologically into the category of vulvar images, many examples of which were recovered during excavations at the beginning of the 20th century at Abri Castanet and the adjacent site of Abri Blanchard. This new discovery from Castanet surely provides an age estimate for those earlier finds, all of which were located within a few meters of the image described here. 


\section{INDEX}

Keywords : Rock shelter Castanet, Engraved block, Context, Datation, Aurignacian

Mots-clés : Abri Castanet, bloc gravé, contexte, datation, Aurignacien

\section{AUTEURS}

\section{ROMAIN MENSAN}

Université Toulouse II-le Mirail, Maison de la Recherche, Laboratoire TRACES UMR 5608, 5, allées Antonio Machado, 31058 Toulouse Cedex 9, France - mensrom@gmail.com

\section{RAPHAËLLE BOURRILLON}

Université Toulouse II-le Mirail, Maison de la Recherche, Laboratoire TRACES UMR 5608 - CREAP, 5, allées Antonio Machado, 31058 Toulouse Cedex 9, France ou Center for the Study of Human Origins, New York University, 25 Waverly Place, New York, NY 10003 USA - bourrillon@univtlse2.fr

\section{CATHERINE CRETIN}

Centre National de Préhistoire, Sous-direction de l'Archéologie, Direction générale des Patrimoines, Ministère de la Culture et de la Communication, 38, rue du 26e Régiment d'Infanterie, 24000 Périgueux, France - catherine.cretin@culture.gouv.fr

\section{RANDALL WHITE}

Center for the Study of Human Origins, New York University, 25 Waverly Place, New York, NY 10003 USA - randall.white@nyu.edu

\section{PHILIPPE GARDÈRE}

Institut National de Recherches Archéologiques Préventives (INRAP), Base de Tours, 148, avenue Maginot, 37000 Tours, France - philippe.gardere@numericable.fr

\section{LAURENT CHIOTTI}

Muséum national d'histoire naturelle, Département de Préhistoire, UMR 7194, abri Pataud, 20, rue du Moyen-Âge, 24620 Les Eyzies-de-Tayac, France - lchiotti@mnhn.fr

\section{MATTHEW SISK}

IDPAS, Department of Anthropology, Stony Brook University, Stony Brook, NY 11794, USA mlsisk@gmail.com

\section{AMY CLARK}

School of Anthropology, PoBox 210030, University of Arizona, Tucson AZ 85721 USA aeclark@email.arizona.edu

\section{THOMAS HIGHAM}

Radiocarbon Accelerator Unit, Research Laboratory for Archaeology \& the History of Art, Dyson Perrins Building, University of Oxford, South Parks Rd, Oxford, OX1 3QY, UK thomas.higham@rlaha.ox.ac.uk 


\section{ÉLISE TARTAR}

UMR 7041 ArScAn, Ethnologie préhistorique, Maison de l'Archéologie et de l'Ethnologie, 21, allée de l'Université - 92023 Nanterre cedex, France - elise.tartar@gmail.com 